\title{
Política Municipal de Saneamento Básico e a ocorrência de doenças nos municípios brasileiros
}

\author{
Municipal Policy of Basic Sanitation and the occurrence of \\ diseases in Brazilian municipalities
}

Natássia Molina Bayer [a] [D, Paulo Ricardo Ricco Uranga [a] [D] Adelar Fochezatto [a] (iD)

[a] Pontifícia Universidade Católica do Rio Grande do Sul (PUC-RS), Porto Alegre, RS, Brasil

Como citar: Bayer, N. M., Uranga, P. R. R., \& Fochezatto, A. (2021). Política Municipal de Saneamento Básico e a ocorrência de doenças nos municípios brasileiros. urbe. Revista Brasileira de Gestão Urbana, v. 13, e20190375. https://doi.org/10.1590/2175-3369.013.e20190375

\section{Resumo}

O saneamento básico inadequado pode ocasionar uma série de doenças endêmicas e epidêmicas. 0 objetivo é analisar a influência da Política Municipal de Saneamento Básico (PMSB) na ocorrência de doenças relacionadas ao saneamento básico inadequado nos municípios brasileiros. Utilizando dados da Pesquisa de Informações Básicas Municipais (MUNIC) de 2017, a análise empírica é dividida em duas etapas. Primeiro, utiliza-se o modelo probit espacial para identificar se a presença da PMSB influencia a probabilidade dos municípios apresentarem doenças. Segundo, utiliza-se o modelo logit para verificar as chances de ocorrência de doenças, levando em consideração o tipo de cluster espacial de PMSB que os municípios pertencem. Os resultados da primeira etapa detectaram a presença de dependência espacial e não permitem afirmar que possuir a PMSB diminui a probabilidade de ocorrência de doenças. Os resultados da segunda etapa mostraram que há uma diminuição das chances de ocorrência de doenças em municípios que pertencem a clusters do tipo HH (municípios com PMSB, cercados por municípios com PMSB) e LH (municípios sem PMSB, cercados por municípios com PMSB). Conclui-se que para a PMSB ser efetiva na redução da ocorrência de doenças, ela deve ser planejada em um contexto espacial mais amplo que os limites municipais.

Palavras-chave: Saneamento básico. Doenças. Probit espacial. Logit.

\section{Abstract}

Inadequate sanitation can lead to several endemic and epidemic diseases. The objective is to analyze the influence of the Municipal Basic Sanitation Policy (PMSB) on the occurrence of diseases related to inadequate basic sanitation in Brazilian municipalities. Using data from the 2017 Municipal Basic Information Survey (MUNIC), the empirical analysis is divided into two steps. First, the spatial probit model is used to identify whether the presence of PMSB influences the likelihood of municipalities having diseases. Second, the logit model is used to verify the chances of diseases occurrence, taking into account the type of PMSB spatial cluster that the

NMB é economista, mestra em Economia do Desenvolvimento, e-mail: natassia.bayer@acad.pucrs.br PRRU é economista, doutorando em Economia do Desenvolvimento, e-mail: paulo.uranga@acad.pucrs.br AF é engenheiro agrônomo, doutor em Economia, e-mail: adelar@pucrs.br 
municipalities belong to. The results of the first stage detected the presence of spatial dependence and do not confirmed that having the PMSB reduces the probability of diseases occurrence in the municipalities. The results of the second stage showed that there is a decreased chance of disease occurrence in municipalities that belong to HH clusters (municipalities with PMSB, surrounded by municipalities with PMSB) and LH clusters (municipalities without PMSB, surrounded by municipalities with PMSB). These results show that PMSB must be planned considering a wider context than municipal boundaries.

Keywords: Basic sanitation. Diseases. Spatial probit. Logit.

\section{Introdução}

A preocupação com o saneamento e sua relação com a saúde humana está presente desde os povos mais antigos. No entanto, foi a partir da Revolução Industrial que os problemas de saúde decorrentes das más práticas sanitárias se intensificaram. Foi necessária uma mudança de comportamento em relação aos hábitos de higiene para conter a propagação de epidemias (Freitas, 2003; Siqueira \& Moraes, 2009).

No Brasil, ocorreram importantes avanços na área de saneamento na primeira metade do século XX, com a atuação do médico sanitarista Oswaldo Cruz. No comando da saúde pública do governo federal, buscou erradicar o surto de doenças causadas por diferentes agentes patológicos. A cidade do Rio de Janeiro recebeu limpeza de ruas, praças, casarões e terrenos, visando eliminar locais criadouros de roedores e insetos, principais transportadores de doenças infectocontagiosas. Nessa época, também foi importante a atuação do sanitarista Saturnino de Brito, que realizou várias obras de saneamento, como os sistemas de distribuição de águas e coleta de esgoto em algumas capitais brasileiras (Vieira, 2017).

O Brasil ainda precisa ampliar o acesso aos serviços de saneamento para alcançar o objetivo estipulado pela ONU (2015), de "assegurar a disponibilidade e a gestão sustentável da água e saneamento para todos". Os dados da Pesquisa Nacional por Amostra de Domicílios (PNAD) de 2015 (IBGE, 2016b) apontam que: $85 \%$ das residências no país eram atendidas pela rede geral de abastecimento de água; a coleta de esgoto sanitário atendia em torno de $64 \%$ dos domicílios; e a coleta de resíduos sólidos atendia cerca de $90 \%$ das unidades domiciliares brasileiras.

0 avanço no marco regulatório do saneamento no país é marcado pela promulgação da Lei 11.445/2007. Esta lei determina as diretrizes nacionais de saneamento e a política federal de saneamento, visando garantir que a prestação destes serviços cumpra os princípios de universalização de acesso, assegurando a saúde da população e a proteção ao meio ambiente. Ela define o saneamento básico como: abastecimento de água; coleta de esgoto sanitário; limpeza urbana e manejo de resíduos sólidos; e drenagem e manejo das águas pluviais do espaço urbano. Estabelece que o titular dos serviços será o responsável por formular e implementar a PMSB e o Plano Nacional de Saneamento Básico (Plansab).

Como já exposto, o saneamento é crucial para a saúde pública, uma vez que pode prevenir os casos de doenças que possuem relação direta com as condições de saneamento inadequado. Hoje grande parcela da população vive em lugares em que as circunstâncias sanitárias são inadequadas, como nas encostas dos morros e nas margens de rios, ficando vulneráveis a contrair doenças infectocontagiosas, gerando custos para a saúde pública (de Menezes \& Uchoa, 2011).

Dada a importância do saneamento para a saúde pública, este trabalho tem como objetivo analisar a influência da PMSB na ocorrência de doenças relacionadas ao saneamento inadequado nos municípios brasileiros. Utilizando informações da MUNIC de 2017 (IBGE, 2018), a análise empírica é dividida em duas etapas. Primeiro, utiliza-se um modelo probit espacial, para verificar se a presença da PMSB influencia a probabilidade de os municípios apresentarem doenças. $O$ uso deste modelo se justifica, pois a ocorrência de endemias e epidemias geralmente apresentam dependência espacial, podendo influenciar a efetividade da PMSB. Segundo, utiliza-se um modelo logit para verificar as chances de ocorrência de doenças nos municípios em função do tipo de cluster espacial de PMSB a que os mesmos pertencem. 
Este estudo está dividido em cinco seções, incluindo esta introdução. Na próxima seção é feita uma revisão de literatura sobre a política de saneamento, as doenças relacionadas ao saneamento e sobre estudos relacionados ao tema. Na sequência, é apresentada a metodologia. Posteriormente apresenta-se a análise dos resultados. Finalmente, são apresentadas as considerações finais.

\section{Revisão bibliográfica}

Nas últimas décadas o saneamento ambiental ganhou espaço no debate das agendas mundiais de desenvolvimento sustentável. Diversas conferências mundiais abordaram a importância de preservar os recursos naturais, garantindo o direito da atual e das futuras gerações viverem com saúde e ambiente limpo. Para a Organização Mundial de Saúde (WHO, 2018), o saneamento é um mecanismo de controle do espaço físico em que o indivíduo está inserido, no qual pode exercer influências negativas sobre o bem-estar físico, mental e social do ser humano (Heller, 1998). De outro modo, o saneamento pode ser considerado um conjunto de métodos que tem por finalidade melhorar o bem-estar da vida das pessoas.

O investimento em saneamento no Brasil foi mais efetivo no período de 1970-80 (Leoneti et al., 2011). No período, houve uma centralização administrativa das empresas que operavam no setor de saneamento, tornando os municípios apenas condutores das políticas do governo federal. A verba deste setor começou a ser administrada pelo Banco Nacional de Habitação, por meio do Plano Nacional de Saneamento (Planasa), criado em 1971. O plano obteve êxito na ampliação do acesso aos serviços de saneamento, mas não conseguiu suprir as demandas decorrentes do processo de urbanização ocorrido naquela época (Turolla, 1999). A paralisação na expansão do setor ocorreu devido à redução dos recursos por causa da crise de 1980 e o Planasa foi extinto em 1992, acarretando um problema institucional regulatório no setor (Scriptore, 2010).

Na Constituição Federal de 1988, o setor de saneamento passa a ser de responsabilidade dos municípios, com algumas atribuições à União e aos Estados, definindo de maneira ambígua onde especificamente cada ente federativo poderia atuar (Scriptore, 2010). Com sanção da lei da Política Nacional de Saneamento Básico (Lei 11.445 de 2007), encerrou-se o período de incertezas sobre o marco legal, dando início uma nova etapa na gestão dos serviços públicos de saneamento (Borja, 2014). No artigo 52 desta mesma lei, fica definido que a União é responsável pela implementação do Plansab, o qual deve abranger todos os serviços de saneamento, cumprindo as diretrizes e metas trazidas na lei com um prazo de vinte anos. Com a promulgação da lei, os governos municipais passam a ter obrigatoriedade de elaborar a PMSB. Foi somente após esse plano que os municípios tiveram a possibilidade de receber verbas do governo federal para investimentos em saneamento (Venson et al., 2017).

A PMSB contém diversas informações sobre as definições, os princípios e os instrumentos para aplicação da lei. A política define o planejamento e a execução de todas as ações na prestação do serviço público, bem como as normas administrativas de regulação e fiscalização. Além disso, especifica questões referentes ao controle social, ao prestador dos serviços públicos de saneamento e as formas de subsídios. Os instrumentos da PMSB serão executados por meio do Plano Municipal de Saneamento, pelo Controle Social, pelo Sistema de Gestão do Saneamento Básico, pelo Fundo Municipal de Saneamento Básico, entre outros (Funasa, 2014).

A Lei federal e as políticas municipais têm como princípios fundamentais a universalização e a prestação dos serviços de saneamento de forma adequada, visando garantir saúde para a população. Sendo assim, é importante identificar quais enfermidades são oriundas de saneamento inadequado. Para distinguir as doenças associadas ao saneamento, Cairncross \& Feachem (1993) criaram uma classificação para patologias infectocontagiosas, que passou a ser utilizada pela Fundação de Nacional da Saúde (Funasa, 2013), nomeando-as de doenças relacionadas ao saneamento ambiental inadequado. Estas podem ter relação com o saneamento inadequado por abastecimento de água não tratada, esgotamento sanitário inadequado, contaminação pela presença de resíduos sólidos ou condições precárias de habitação, sendo 
divididas em cinco categorias: doenças de transmissão feco-oral por ingestão ou contato com a água (diarreia, febres entéricas, hepatite A); doenças transmitidas por inseto vetor (dengue, febre amarela, leishmanioses, filariose linfática, malária, doença de chagas); doenças transmitidas através do contato com a água (esquistossomose, leptospirose); doenças relacionadas com a higiene (doenças dos olhos, tracoma, conjuntivites, doenças da pele, micoses superficiais); e geo-helmintos e teníases (helmintíases, teníases).

Existem, portanto, inúmeras doenças provocadas pela falta de saneamento apropriado. Outros elementos podem estar associados ao aparecimento destas patologias, como a ausência de esgotamento sanitário e o acondicionamento e destinação final dos resíduos sólidos. As melhorias nas condições do saneamento ambiental seriam capazes de diminuir a incidência de doenças em crianças e na população idosa, considerados grupos mais frágeis e suscetíveis a contrair esse tipo de enfermidade (Cutler \& Miller, 2005; Esrey \& Habicht, 1986; Silva \& Esperidião, 2017).

Nos países em desenvolvimento, como o Brasil, as doenças causadas por problemas no saneamento, principalmente o contágio por veiculação hídrica, é bastante recorrente na área da saúde pública. Venson et al. (2017) realizaram uma pesquisa para os municípios baianos no período de 2006 a 2012, buscando identificar a evolução da distribuição espacial no acesso à água e coleta de esgoto, a fim de verificar as implicações da Lei de Saneamento Básico de 2007. Por meio do método de Análise Exploratória dos Dados Espaciais (AEDE) foi possível observar um atendimento muito concentrado em regiões mais populosas e com maiores níveis de renda per capita, diferentemente das áreas menos densas e mais pobres em que apresentavam carência no acesso aos serviços de saneamento.

Com o objetivo de avaliar como o atendimento do saneamento impacta a saúde da população brasileira Uhr et al. (2016) analisam as internações hospitalares por doenças ocasionadas pelo consumo de água contaminada e o contato com a pele, além da coleta sanitária inadequada no período de 2000 a 2011. Os dados apontam que o crescimento no acesso ao saneamento reduz o número de casos de internações, sobretudo o acesso ao sistema de esgotamento sanitário.

Teixeira et al. (2014) afirmou que no Brasil, o número de óbitos ocasionados pela oferta inadequada de saneamento resultou em mais de 13 mil no período de 2001 a 2009. Paiva \& Souza (2018) avaliaram a relação entre as condições de saneamento, atendimento das equipes de saúde da família e as variáveis socioeconômicas sobre as enfermidades causadas pela água não tratada no Brasil. Usando dados da PNAD e DATASUS os autores estimaram que cerca de $16 \%$ das internações causadas por veiculação hídrica poderiam ser evitadas se as condições da rede coletora de esgoto fossem apropriadas.

Para verificar as correlações entre índices de saúde e saneamento no Brasil, Mendonça \& Motta (2007) estimaram um modelo com dados em painel usando dados dos censos de 1980, 1991 e 2000. Através desse método foi possível observar uma redução na mortalidade infantil decorrente de doenças de veiculação hídrica, devido a melhora no acesso ao saneamento e também a expansão no acesso aos serviços de educação e saúde. Diante dos resultados, calcularam a média referente ao custo de salvar uma vida e constataram que os custos com a melhora na cobertura dos serviços de saneamento e a diminuição do analfabetismo são alternativas para diminuir a ocorrência de óbitos por veiculação hídrica, sendo a redução do analfabetismo o meio mais barato.

Araújo et al. (2010) analisam a eficiência dos serviços de saneamento nos municípios de Tocantins na redução de enfermidades no ano de 2006. Para isso, os autores analisaram um grande número de patologias, bem como o modo que elas foram transmitidas, o total de internações hospitalares e a variável coleta de lixo, utilizando a metodologia da Análise Envoltória de Dados (DEA). Neste trabalho foi possível observar os benefícios do acesso aos serviços de saneamento, realizando uma comparação e identificação dos municípios considerados eficientes e ineficientes no combate às epidemias.

Teixeira (2011) avalia se os índices de saneamento impactam no número de óbitos e internações hospitalares por intervalos de idade e tipos de patologias nos municípios brasileiros no período de 2001 a 2008. Através da aplicação metodológica de dados em painel desbalanceado, os resultados apontaram que a diminuição na presença de coliformes totais impactaria na redução no número de mortes de crianças menores de 1 ano. Além disso, o aumento no abastecimento de água e diminuição nos coliformes totais 
reduzem o número de morbidades para os casos de disenteria, tuberculose e hepatite. 0 esgotamento sanitário reduz a mortalidade para crianças de até 4 anos e internações nas crianças de 5 a 9 anos.

Okawara (2018) verifica se o gerenciamento dos resíduos sólidos nos municípios brasileiros influencia os indicadores de saúde pública. 0 autor faz uma avaliação do impacto da Lei Nacional de Resíduos Sólidos (Lei 12.305) na produção de lixo e na presença de lixões para o intervalo de 2008 a 2015, buscando identificar a ocorrência de óbitos de crianças e morbidade devido às doenças ocasionadas pelos resíduos sólidos (diarreia, febre tifoide, malária, entre outras). Os resultados confirmam que os Planos Municipais de Gerenciamento de Resíduos Sólidos não são suficientes para diminuir a produção de lixo e também o encerramento das atividades dos lixões (metas definidas na PNRS). Entretanto, as evidências apontaram que a efetuação de tais políticas municipais é importante para a redução no número de casos de Leptospirose.

Em estudo realizado para o nordeste do país, foram coletados dados do IPEADATA e do PNUD para 1991, 2000 e 2010 referentes ao fornecimento de água e coleta de esgoto e variáveis como PIB per capita, percentual da população com 18 anos ou mais com fundamental completo, Índice de Gini, Índice de Desenvolvimento Humano (IDH) e despesas com saúde e saneamento, as quais correspondem à situação socioeconômica do território nordestino. De acordo com os resultados encontrados, percebe-se que a precariedade dos serviços de saneamento é diretamente relacionada à mortalidade infantil e com a problemática socioeconômica. As áreas com indicadores inapropriados de saneamento são os espaços onde mais ocorrem óbitos de crianças. A melhora nos índices educacionais proporciona maior demanda aos serviços de abastecimento de água e coleta de esgoto adequados, além da redução da desigualdade socioeconômica (Silva \& Esperidião, 2017).

Conforme as pesquisas apresentadas, tanto a ampliação dos serviços de abastecimento de água, a coleta e tratamento de esgoto sanitário, a limpeza urbana e o manejo de resíduos sólidos quanto a drenagem e o manejo das águas pluviais do espaço urbano, colaboram para o desenvolvimento econômico do país e para a diminuição dos impactos ambientais. 0 acesso ao saneamento adequado também é efetivo na redução dos casos de mortalidade e morbidade, diminuindo os gastos em assistência médica e sanitária com as doenças. Na próxima seção será apresentada a metodologia empregada para avaliar o efeito de diferentes variáveis na ocorrência de endemias e/ou epidemias de doenças associadas ao saneamento.

\section{Metodologia}

Os dados utilizados são da MUNIC, uma pesquisa realizada pelo Instituto Brasileiro de Geografia e Estatística (IBGE) que contêm informações sobre habitação, transporte, meio ambiente, gestão de riscos, entre outros. Para este trabalho foi utilizada a base de dados da MUNIC de 2017 (IBGE, 2017b), contendo respostas de todas as 5.570 prefeituras existentes no país. Tanto a organização dos dados quanto os métodos empregados foram elaborados e processados no software R.

O Quadro 1 apresenta a descrição das variáveis utilizadas nesse estudo. Além da variável dependente, como explicativas serão utilizadas variáveis retiradas do IBGE (2016a; 2017a; 2017b) e FIRJAN (2016). Para a densidade populacional e o PIB per capita foi aplicado o logaritmo natural, pois apresentam valores muito maiores que a as variáveis dependentes binárias. Assim como para o PIB per capita, para calcular a taxa de urbanização foi utilizada a população estimada para os anos de 2016, e para a densidade populacional a estimativa de 2017. Como proxy para a educação no município se utilizou o indicador municipal da FIRJAN para a educação, que emprega, entre outros dados, o atendimento à educação infantil e o abandono, a distorção idade-série e o resultado no IDEB no Ensino Fundamental. Há nos modelos variáveis relativas à existência: de favelas, cortiços e loteamentos irregulares; de impactos ambientais em corpos d'água nos últimos 24 meses; de alagamentos, enchentes ou enxurradas nos últimos 4 anos; e da variável objeto desse estudo, a PMSB. 
Quadro 1 - Descrição das variáveis utilizadas nos modelos

\begin{tabular}{|c|c|c|}
\hline Variáveis & Descrição & Fonte \\
\hline \multirow{2}{*}{ end_epi } & A prefeitura tem conhecimento da ocorrência de endemia(s) ou epidemia(s) & \multirow{2}{*}{ IBGE } \\
\hline & associada(s) ao saneamento básico, nos últimos 12 meses, MUNIC/2017 (Sim=1; Não=0) & \\
\hline Indens 17 & Logaritmo natural da densidade populacional (hab/km2) (2017) & IBGE \\
\hline †xurbl6 & Taxa de urbanização (2016) & IBGE \\
\hline Inpibpcl6 & Logaritmo natural do PIB per capita (2016) & IBGE \\
\hline ifdml beduc & Indicador de educação (2016) & FIRJAN \\
\hline favelas & Existem no município favelas, cortiços e loteamentos irregulares, MUNIC/2017 (Sim=1; Não=0) & IBGE \\
\hline imp_polagua & (*) Polvição de algum corpo d'água, MUNIC/2017 (Sim=1; Não=0) & IBGE \\
\hline imp_assagua & (*) Assoreamento de algum corpo d'água, MUNIC/2017 (Sim=1; Não=0) & IBGE \\
\hline imp_dimagua & (*) Diminuição de vazão de algum corpo d'água, MUNIC/2017 (Sim=1; Não=0) & IBGE \\
\hline imp_esgoto & $\begin{array}{l}\text { (*) Falta de saneamento (destinação inadequada do esgoto doméstico), } \\
\text { MUNIC/2017 ( } \operatorname{Sim}=1 ; N a ̃ o=0)\end{array}$ & IBGE \\
\hline alagamento & O município foi atingido por alagamentos nos últimos 4 anos, MUNIC/2017 (Sim=1; Não=0) & IBGE \\
\hline enchente & $\begin{array}{l}\text { O município foi atingido por enchentes ou inundações graduais nos últimos } 4 \text { anos, } \\
\text { MUNIC/2017 ( } \operatorname{Sim}=1 ; \mathrm{Não}=0 \text { ) }\end{array}$ & IBGE \\
\hline enxurrada & $\begin{array}{l}\text { O município foi atingido por enxurradas ou inundações bruscas nos últimos } 4 \text { anos, } \\
\text { MUNIC/2017 (Sim=1; Não=0) }\end{array}$ & IBGE \\
\hline pol_sanbas & PMSB - existência, MUNIC/2017 (Sim=1; Não=0) & IBGE \\
\hline
\end{tabular}

Fonte: Elaboração própria. (*) Observação no município da ocorrência de algum impacto ambiental e/ou processo/ação que resulte em impacto no ambiente nos últimos 24 meses.

A Tabela 1 apresenta as estatísticas descritivas das variáveis utilizadas neste estudo. É possível verificar que $34,7 \%$ dos municípios reportaram ter casos de endemias e/ou epidemias em 2017. Os municípios em média apresentavam uma densidade populacional de 117 habitantes por $\mathrm{km}^{2} \mathrm{e}$ o máximo de 13.715,6. A média do PIB per capita corresponde a 21.126,2 reais em 2016. 0 índice da educação no país era de 0,769. Em relação à variável de ouvidoria, somente $31,4 \%$ dos municípios possuíam um canal de comunicação entre a população e as prefeituras em 2017. A variável favelas mostrou que 67,6\% dos municípios tinham alguma dessas irregularidades, comprovando que não existia uma estrutura urbana segura à população, estando exposta a riscos ambientais, de saúde, entre outros. A PMSB foi adotada por apenas 38,2\% dos municípios brasileiros, comprovando o atraso na efetivação e ampliação no acesso ao serviços de saneamento.

Tabela 1 - Estatísticas descritivas das variáveis utilizadas nos modelos.

\begin{tabular}{lllll}
\hline Variáveis & Média & Desvio Padrão & Mínimo & Máximo \\
\hline end_epi & 0,347 & 0,476 & 0,000 & 1,000 \\
dens17 & 117,838 & 615,788 & 0,075 & $13.715,620$ \\
Indens17 & 3,270 & 1,435 & $-2,586$ & 9,526 \\
txurb16 & 0,638 & 0,220 & 0,042 & 1,000 \\
pibpc16 & $21,126,180$ & $20.332,650$ & $3.190,570$ & 12,659 \\
Inpibpc16 & 9,697 & 0,683 & 8,068 & 1,000 \\
ifdm16educ & 0,769 & 0,114 & 0,383 & 1,000 \\
ouvi_sanbas & 0,314 & 0,464 & 0,000 & 1,000 \\
Favelas & 0,676 & 0,468 & 0,000 & 1,000 \\
imp_polagua & 0,153 & 0,360 & 0,000 & 1,000 \\
imp_assagua & 0,146 & 0,353 & 0,000 & 1,000 \\
imp_dimagua & 0,201 & 0,400 & 0,000 & 1,000 \\
imp_esgoto & 0,249 & 0,432 & 0,000 & 1,000 \\
Alagamento & 0,310 & 0,463 & 0,000 & 1,000 \\
Enchente & 0,272 & 0,445 & 0,000 & 1,000 \\
Enxurrada & 0,285 & 0,452 & 0,000 & 1,000 \\
pol_sanbas & 0,382 & 0,486 & 0,000 & \\
\hline
\end{tabular}

Fonte: Elaboração própria. 
No Brasil, em 1.935 municípios as prefeituras notificaram a ocorrência de endemias e/ou epidemias de doenças associadas à falta de saneamento. A Figura 1 apresenta um mapa dos municípios brasileiros, indicando aqueles em que as prefeituras reportaram ter conhecimento da existência de alguma das epidemias e/ou endemias (cor vermelha) e aqueles em que as prefeituras reportaram não ter conhecimento da incidência das mesmas (cor cinza). Assim, o mapa fornece uma visão panorâmica da distribuição espacial das doenças associadas ao saneamento nos municípios brasileiros em 2017.

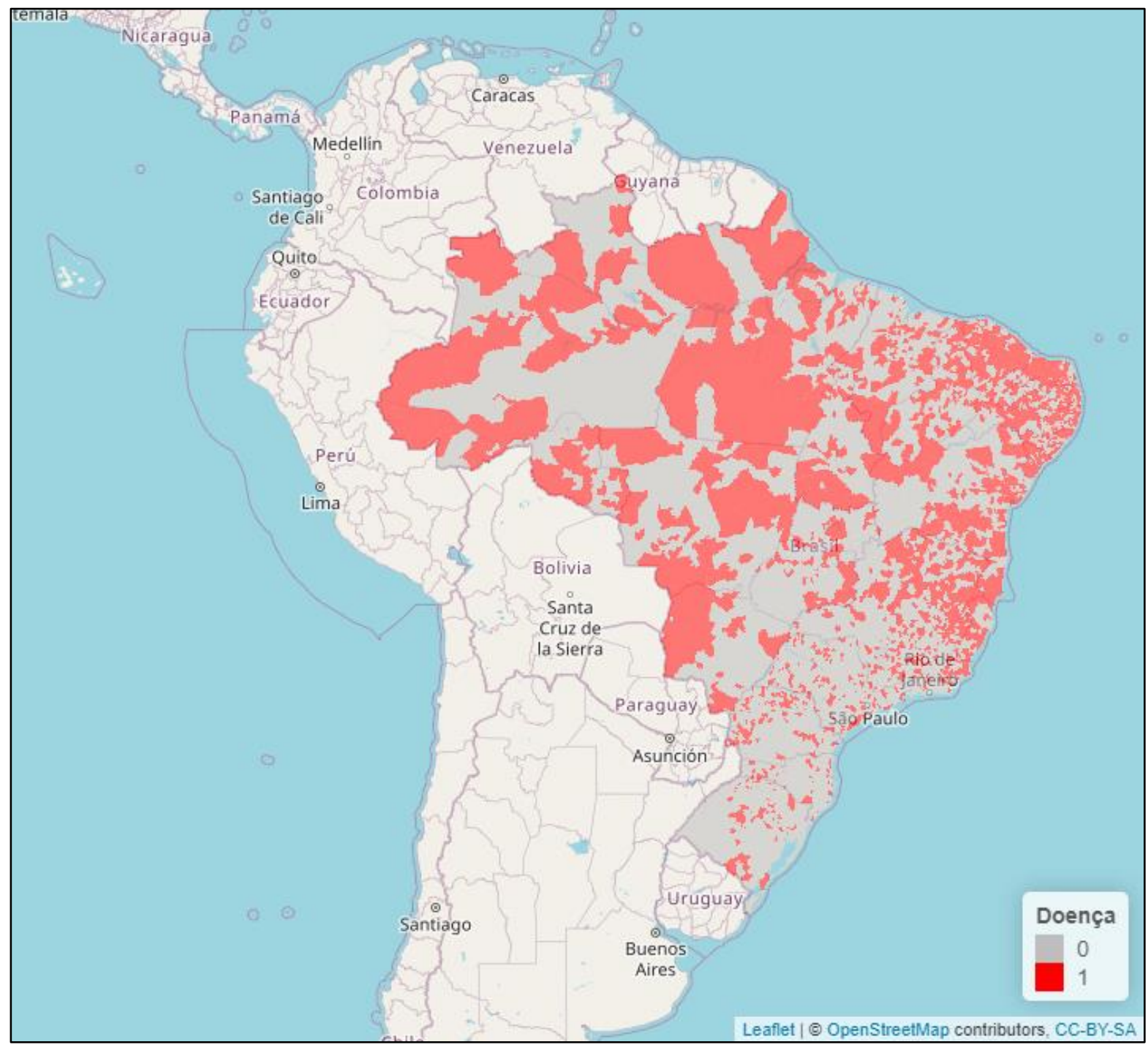

Figura 1 - Mapa das cidades brasileiras que apresentaram epidemias e/ou endemias associadas ao saneamento básico nos últimos 12 meses para o ano de 2017. Notas: 0 = A prefeitura não tem conhecimento se teve doença associada ao saneamento no município; 1 = A prefeitura tem conhecimento de que teve doença associada ao saneamento no município. Fonte: Elaboração própria.

Para cumprir o primeiro objetivo deste trabalho, após a identificação dos números de ocorrências de epidemias e/ou endemias, calculou-se a correlação espacial existente para cada doença. A Tabela 2 apresenta esses dados e as correlações para as matrizes de pesos espaciais de contiguidade Queen ${ }^{1}$ e de $\mathrm{k}$ vizinhos mais próximos ( $\mathrm{k}=1,3$ e 5). As epidemias ou endemias mais frequentes foram as de dengue (1.501) e diarreia (1.288), ambas apresentaram alta correlação espacial para 1 vizinho mais próximo, 0,244 e 0,215 respectivamente. Outras doenças resultaram em um alto I de Moran para essa matriz, particularmente a

\footnotetext{
${ }^{1}$ A matriz Queen é a mais utilizada na literatura para explicar a dependência espacial, uma vez que considera os municípios vizinhos todos aqueles que fazem fronteira com a unidade observada.
} 
Chikungunya $(0,311)$, a malária $(0,220)$, as verminoses $(0,217)$ e a Zika $(0,207)$. Verificada a correlação, optou-se por testar 4 modelos probit: não espacial, não espacial com dummies regionais, espacial e espacial com dummies regionais.

Para cumprir o segundo objetivo desse trabalho, investigou-se diferentes clusters relacionados a PMSB e como afetam a probabilidade de o município apresentar doenças decorrentes das más condições de saneamento. Para isso, primeiro foram estimados os clusters espaciais de municípios ${ }^{2}$, tendo sido definidos quatro tipos de clusters: Alto-Alto (HH), Alto-Baixo (HL), Baixo-Alto (LH) e Baixo-Baixo (LL). Posteriormente, foram estimados quatro modelos logit, um para cada cluster de municípios. Nestes, a variável existência de doenças (end_epi) foi utilizada como uma variável explicativa. Assim, um coeficiente negativo associado a essa variável significa que municípios que tiveram doenças têm menor probabilidade de pertencerem ao cluster de municípios em questão e vice-versa. Por exemplo, considerando o cluster HH (o município possui PMSB e todos os seus municípios vizinhos também possuem a política), um sinal negativo para a variável "end_epi" significa que os municípios que apresentaram doenças têm menor probabilidade de estarem ou pertencerem ao cluster HH. A Figura 2 apresenta o fluxograma dos métodos utilizados. A explicação mais detalhada da metodologia está abaixo deste.

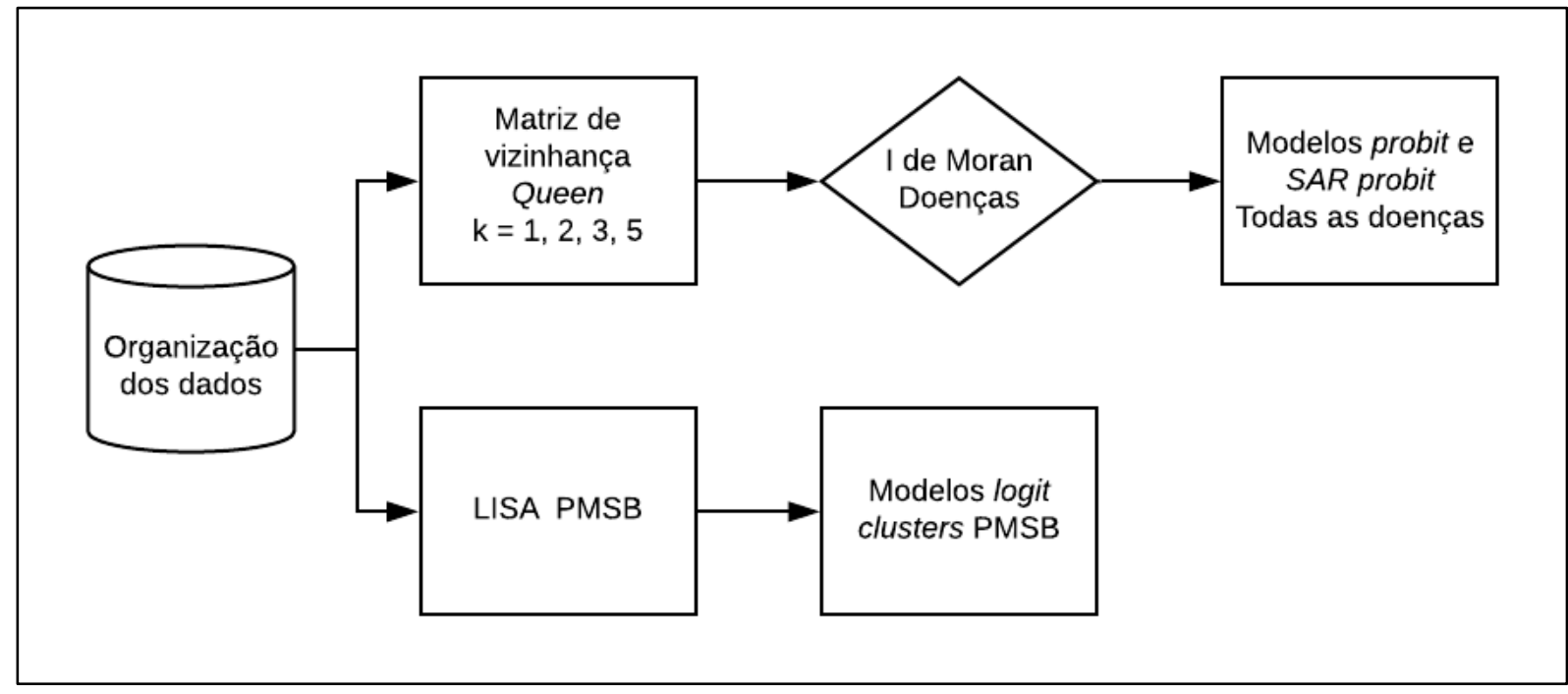

Figura 2 - Fluxograma da metodologia utilizada. Fonte: Elaboração própria.

Altos valores do I de Moran sugerem a utilização de modelos que levem em consideração a dependência espacial. Modelos como o Spatial Autoregressive Model (SAR) são comumente utilizados para verificar a disseminação e riscos de doenças (Lopez \& Manogaran, 2017; Rood et al., 2017; Tipayamongkholgul, 2013), uma vez que epidemias e endemias são fenômenos reconhecidamente regionais e possuem dependência espacial. A especificação do modelo SAR é descrita conforme a equação 1:

$Y=\rho W Y+X \beta+\varepsilon$

Segundo Câmara et al. (2002) o propósito deste modelo é inserir a autocorrelação espacial como uma variável explicativa. Em que $W$ é a matriz de proximidade espacial e a multiplicação de $W Y$ mostra a dependência espacial em $Y$, $\rho$ é o coeficiente espacial autoregressivo e $X$ é um vetor contendo as variáveis

\footnotetext{
2 Para estimar os clusters espaciais foi utilizado o método LISA (Local Indicators of Spatial Association) desenvolvido por Anselin (1995). Outro método de identificação de clusters espaciais é o Gi* (Getis-Ord Gi*) de Getis \& Ord (1992, 1996). A referência clássica sobre econometria espacial é Anselin (1988).
} 
independentes. Quando não há autocorrelação espacial o $\rho=0$, ou seja, é igual a um modelo de regressão tradicional.

Tabela 2 - Número de cidades que reportaram ter conhecimento de epidemias e endemias de doenças associadas ao saneamento básico e I de Moran para diferentes matrizes espaciais.

\begin{tabular}{llllll}
\hline Doenças & Cidades com & I de Moran & & & \\
\cline { 2 - 6 } & ocorrência & Queen & 1 vizinho & 3 vizinhos & $\mathbf{5}$ vizinhos \\
\hline Todas & 1.935 & 0,237 & 0,252 & 0,244 & 0,230 \\
Diarreia & 1.288 & 0,187 & 0,215 & 0,198 & 0,182 \\
Leptospirose & 250 & 0,078 & 0,068 & 0,068 & 0,059 \\
Verminoses & 960 & 0,184 & 0,217 & 0,194 & 0,182 \\
Cólera & 97 & 0,028 & 0,023 & 0,024 & 0,019 \\
Difteria & 163 & 0,033 & 0,071 & 0,036 & 0,023 \\
Dengue & 1.501 & 0,228 & 0,244 & 0,238 & 0,223 \\
Zika & 814 & 0,218 & 0,207 & 0,210 & 0,208 \\
Chikungunya & 958 & 0,305 & 0,311 & 0,307 & 0,269 \\
Tifo & 56 & 0,004 & 0,023 & 0,001 & 0,001 \\
Malária & 181 & 0,201 & 0,220 & 0,218 & 0,198 \\
Hepatite & 428 & 0,074 & 0,091 & 0,080 & 0,076 \\
Febre amarela & 161 & 0,105 & 0,107 & 0,090 & 0,086 \\
Dermatite & 431 & 0,094 & 0,146 & 0,112 & 0,094 \\
Doença respiratória & 650 & 0,126 & 0,151 & 0,145 & 0,128 \\
Outras & 191 & 0,077 & 0,096 & 0,091 & 0,074 \\
\hline
\end{tabular}

Fonte: Elaboração própria.

Por outro lado, a característica dicotômica das variáveis dependentes estudadas (a prefeitura ter ou não conhecimento de endemia ou epidemia) se adéqua a modelos de resposta binária, que se utilizam de funções de distribuição acumulada para restringir as previsões entre 0 e 1 (Gujarati, 2006; Wooldridge, 2014). Usualmente é utilizada a função normal, em modelos probit, conforme as equações 2 e 3 :

$P(Y=1 / X)=F\left(\beta_{0}+\beta X\right)$

$F\left(\beta_{0}+\beta X\right)=\int_{-\infty}^{\beta_{0}+\beta X} \frac{1}{\sqrt{2 \pi}} e^{-\left(\beta_{0}+\beta X\right)^{2} / 2}$

em que $Y$ é a variável binária estudada e $F$ é a função de distribuição acumulada requerida, no caso a função normal, $X$ é o vetor de varáveis explicativas, $\beta_{0}$ é o intercepto e $\beta$ os demais parâmetros estimados.

Em virtude da natureza dos dados, optou-se pela utilização de modelos SAR probit para verificar o efeito das políticas de saneamento e demais variáveis na ocorrência de endemias e epidemias de doenças reportadas pelas prefeituras. Conforme LeSage \& Pace (2009), o modelo pode ser escrito como mostrado na equação 4:

$Y^{*}=\left(I_{n}-\rho W\right)^{-1}(\beta X+\varepsilon)$

em que $X$ é o vetor de varáveis explicativas, $\beta$ os parâmetros estimados, $\rho$ é o coeficiente espacial autoregressivo e $W$ a matriz de pesos espaciais. Nos casos de $\rho=0$ ou $W=I_{n}$ o modelo se iguala a um probit convencional. $Y$ é a variável dependente que tem valor 1 se a prefeitura da cidade reportou endemia e/ou epidemia de doenças ligadas ao saneamento nos últimos 12 meses, e valor 0 caso contrário. $Y^{*}$ é uma variável latente, não observada, que assume os valores preditos de $Y_{i}$ conforme a equação 5:

$Y_{i}=1$ se $Y_{i}^{*} \geq 0$ 
$Y_{i}=0$ se $Y_{i}^{*}<0$

A variável dependente para os modelos probit e SAR probit é a ocorrência de endemia e/ou epidemia de doenças associadas ao saneamento no Brasil para o ano de 2017. Para os modelos SAR probit estimados foi utilizada a matriz de 1 vizinho mais próximo, que apresentou os maiores valores de I de Moran para a variável dependente.

Como já explicado, para cumprir o segundo objetivo deste trabalho foi verificar a existência de clusters espaciais dos municípios que possuem (ou não) PMSB através dos indicadores LISA, usando uma matriz de pesos espaciais do tipo Queen. Os clusters resultantes, HH, HL, LH e LL, foram incluídos em quatro modelos logit como variáveis dependentes, conforme a equação 6 :

$Y^{\text {Cluster }}=\beta_{0}+\beta_{1} X_{\text {end_epi }}+\beta X+\mu$

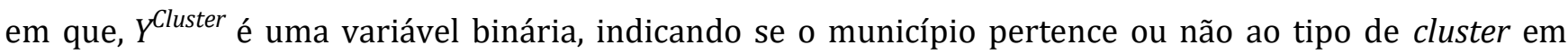
questão (valor $=1$, se pertence e 0 se não pertence); $X_{\text {end_epi }}$ é a variável que indica se o município apresentou endemia ou epidemia de doenças relacionadas ao saneamento nos últimos doze meses (valor = 1 , se apresentou e 0 se não apresentou); e $X$ é o vetor das demais varáveis explicativas.

Na próxima seção serão apresentados os quatro modelos espaciais e não espaciais estimados neste estudo, bem como a separação por grandes regiões do país, visando averiguar se a PMSB influencia a ocorrência de doenças ligadas às condições de saneamento nos municípios do país. Também serão mostrados os resultados dos modelos logit para os clusters de municípios com PMSB.

\section{Análise dos resultados}

Foram utilizados quatro modelos para estimar a influência da PMSB na redução de endemias e epidemias no Brasil. Os coeficientes e os testes dos modelos são apresentados na Tabela 3. Os modelos $1 \mathrm{e}$ 2 são probit não espaciais, enquanto os de número 3 e 4 são probit que levam em consideração a dependência espacial. Nos modelos 2 e 4 foram incluídas dummies das 5 Grandes Regiões brasileiras, sendo a região do Centro-Oeste utilizada como referência nos modelos.

O logaritmo da densidade populacional e as variáveis que indicam se o município teve alagamentos e enchentes ou inundações nos últimos 4 anos não foram significativas em nenhum dos modelos. A proxy para o nível de educação se mostrou importante para diminuir as chances de endemias e epidemias de doenças associadas ao saneamento em todos os modelos.

Ter ouvidoria municipal ou central de atendimento ao cidadão para recebimento de reclamações ou manifestações sobre os serviços de saneamento teve relação positiva com a variável dependente em todos os modelos, bem como a existência de favelas, cortiços e loteamentos irregulares na cidade. A presença das regiões nos modelos 2 e 4 torna o efeito da PMSB não significante. Além disso, o coeficiente espacial autorregressivo foi significante para os modelos SAR probit (3 e 4), o que indica que considerar dependência espacial é um fator importante para explicar a incidência de endemias e epidemias.

As estatísticas de teste dos modelos apontam o último (SAR probit com as Grandes Regiões) como o mais bem ajustado, pois possui o maior logaritmo da verossimilhança $(-3.195,223)$ e segundo menor valor no teste Hosmer \& Lemeshow $(15,354)$, o que possibilita afirmar que se trata de um bom ajuste a $10 \%$. 
Tabela 3 - Coeficientes e testes dos modelos probit não espaciais e espaciais

\begin{tabular}{|c|c|c|c|c|c|c|c|c|}
\hline Variáveis & Modelo 1 & & Modelo 2 & & Modelo 3 & & Modelo 4 & \\
\hline Constante & 3,471 & *** & 0,106 & & 2,931 & $* * *$ & 0,171 & \\
\hline Indens17 & 0,009 & & $-0,002$ & & 0,000 & & $-0,007$ & \\
\hline txurbl 6 & 0,427 & $* * *$ & 0,149 & & 0,390 & $* * *$ & 0,152 & \\
\hline Inpibpc16 & $-0,286$ & $* * *$ & $-0,046$ & & $-0,248$ & $* * *$ & $-0,050$ & \\
\hline ifdml beduc & $-2,194$ & $* * *$ & $-0,794$ & $* * *$ & $-1,774$ & *** & $-0,675$ & $* * *$ \\
\hline ouvi_sanbas & 0,238 & $* * *$ & 0,188 & *** & 0,228 & *** & 0,184 & $* * *$ \\
\hline Favelas & 0,223 & $* * *$ & 0,257 & $* * *$ & 0,217 & *** & 0,252 & $* * *$ \\
\hline imp_polagua & 0,087 & * & 0,084 & & 0,091 & $*$ & 0,083 & \\
\hline imp_assagua & 0,072 & & 0,085 & * & 0,081 & & 0,096 & $*$ \\
\hline imp_dimagua & 0,161 & $* * *$ & 0,102 & ** & 0,138 & *** & 0,094 & $* *$ \\
\hline imp_esgoto & 0,142 & $* * *$ & 0,136 & $* * *$ & 0,140 & *** & 0,135 & $* * *$ \\
\hline Alagamento & 0,023 & & 0,052 & & 0,014 & & 0,039 & \\
\hline Enchente & $-0,016$ & & 0,073 & & $-0,018$ & & 0,062 & \\
\hline Enxurrada & $-0,096$ & * & 0,029 & & $-0,080$ & & 0,025 & \\
\hline pol_sanbas & $-0,106$ & $* * *$ & 0,003 & & $-0,104$ & *** & $-0,003$ & \\
\hline I(REGNAT)N & & & 0,552 & *** & & & 0,464 & $* * *$ \\
\hline I(REGNAT)NO & & & 0,379 & *** & & & 0,309 & $* * *$ \\
\hline I(REGNAT)SE & & & $-0,076$ & & & & $-0,074$ & \\
\hline I(REGNAT)S & & & $-0,627$ & *** & & & $-0,528$ & $* * *$ \\
\hline Rho & & & & & 0,220 & *** & 0,170 & *** \\
\hline $\mathrm{AIC}$ & $6.663,1$ & & $6.436,0$ & & - & & - & \\
\hline LogLik & $-3.316,567$ & & $-3.198,978$ & & $-3.297,022$ & & $-3.195,223$ & \\
\hline Pseudo-R2 & 0,078 & & 0,111 & & & & - & \\
\hline Hosmer and Lemeshow & 46,197 & *** & 12,818 & & 42,049 & $* * *$ & 15,354 & * \\
\hline Correctly classified & $66,57 \%$ & & $68,90 \%$ & & $66,80 \%$ & & $68,76 \%$ & \\
\hline Sensitivity & $26,98 \%$ & & $41,40 \%$ & & $28,11 \%$ & & $41,81 \%$ & \\
\hline Specificity & $87,65 \%$ & & $83,55 \%$ & & $87,40 \%$ & & $83,11 \%$ & \\
\hline Area under ROC curve & 0,691 & & 0,723 & & 0,697 & & 0,724 & \\
\hline
\end{tabular}

Fonte: Elaboração própria. Obs: ${ }^{*} p=0,10,{ }^{* *} p=0,05,{ }^{* * *} p=0,01$.

Entretanto foi o Modelo 4 que apresentou maior sensitividade (acertos nos casos de cidades com endemia e epidemia). A comparação desses últimos testes não permite identificar se o trade-off entre sensitividade e especificidade faz o modelo ter um maior poder discriminatório (Bradley, 1997). Nesse caso, a área sob a curva ROC (Receiver Operating Characteristic), que leva em consideração as classificações corretas para 0 e 1 , deve ter o maior valor possível. 0 Modelo 4 apresentou valor 0,724 , sendo o que melhor discriminou entre todos os testados, sendo este o escolhido para o restante da análise.

O impacto das variáveis explicativas na ocorrência de endemias e epidemias de doenças associadas ao saneamento pode ser verificado na Tabela 4. Os efeitos marginais diretos dizem respeito à média de efeitos de todas as observações na mudança de uma variável sobre uma única observação. Já os efeitos indiretos usam essa mesma média sobre a probabilidade de escolha das observações vizinhas. Os efeitos totais são a soma dos efeitos diretos e indiretos. Em outras palavras, seja $\operatorname{Sr}(W)$ uma matriz de vizinhança que captura os impactos de todas as observações, os efeitos totais seriam a soma de todas as colunas da matriz, os efeitos diretos seriam a diagonal e os efeitos indiretos a subtração dos efeitos totais pelos diretos (Wilhelm \& Matos, 2013; Martinetti \& Geniaux, 2017).

Os efeitos diretos foram, em média, responsáveis por $83,7 \%$ da explicação dos efeitos totais, enquanto coube aos efeitos indiretos explicar o restante $(16,3 \%)$. Esses resultados afirmam a importância de se considerar os efeitos dos vizinhos quando detectada a autocorrelação espacial do objeto de estudo. Para os três efeitos marginais, as variáveis significativas resultaram nas mesmas identificadas nos coeficientes do Modelo 4 da Tabela 3. 
Tabela 4 - Efeitos marginais do Modelo SAR probit com as Grandes Regiões brasileiras

\begin{tabular}{|c|c|c|c|c|c|c|}
\hline \multirow[b]{2}{*}{ Variáveis } & \multicolumn{6}{|c|}{ Efeitos Marginais } \\
\hline & Diretos & & Indiretos & & Totais & \\
\hline Indens17 & $-0,002$ & & 0,000 & & $-0,003$ & \\
\hline txurbl 6 & 0,049 & & 0,010 & & 0,059 & \\
\hline Inpibpc16 & $-0,016$ & & $-0,003$ & & $-0,020$ & \\
\hline ifdml6educ & $-0,220$ & $* * *$ & $-0,043$ & $* * *$ & $-0,262$ & $* * *$ \\
\hline ouvi_sanbas & 0,060 & $* * *$ & 0,012 & $* * *$ & 0,071 & $* * *$ \\
\hline Favelas & 0,082 & $* * *$ & 0,016 & $* * *$ & 0,098 & $* * *$ \\
\hline imp_polagua & 0,027 & & 0,005 & & 0,032 & \\
\hline imp_assagua & 0,031 & * & 0,006 & * & 0,038 & $*$ \\
\hline imp_dimagua & 0,031 & $* *$ & 0,006 & $* *$ & 0,036 & $* *$ \\
\hline imp_esgoto & 0,044 & $* * *$ & 0,009 & $* * *$ & 0,052 & $* * *$ \\
\hline Alagamento & 0,013 & & 0,003 & & 0,015 & \\
\hline Enchente & 0,020 & & 0,004 & & 0,024 & \\
\hline Enxurrada & 0,008 & & 0,002 & & 0,010 & \\
\hline pol_sanbas & $-0,001$ & & 0,000 & & $-0,001$ & \\
\hline I(REGNAT)N & 0,151 & $* * *$ & 0,029 & $* * *$ & 0,180 & $* * *$ \\
\hline I(REGNAT)NO & 0,101 & $* * *$ & 0,020 & $* * *$ & 0,120 & $* * *$ \\
\hline I(REGNAT)SE & $-0,024$ & & $-0,005$ & & $-0,029$ & \\
\hline I(REGNAT)S & $-0,172$ & $* * *$ & $-0,033$ & $* * *$ & $-0,205$ & $* * *$ \\
\hline
\end{tabular}

Nota: Esta Tabela contém os resultados dos efeitos marginais oriundos do Modelo 4, apresentados na Tabela 3. Fonte: Elaboração própria. Obs: ${ }^{*} p=0,10,{ }^{* *} p=0,05,{ }^{* * *} p=0,01$.

A densidade populacional não foi significativa e não apresentou efeito marginal no modelo analisado. A taxa de urbanização e o PIB per capita perderam a significância com a inclusão das regiões, assim como a ocorrência de enxurradas. Possivelmente as características regionais incorporaram aspectos socioeconômicos e climáticos que impactam na ocorrência de doenças ligadas ao saneamento inadequado.

A existência da PMSB no município também não teve efeito na presença das Grande Regiões. A inclusão dessas variáveis representa que as características regionais podem ter grande influência sobre a probabilidade de ocorrência ou não endemias e epidemias, e a simples existência da política não supera as dificuldades regionais.

A proxy para educação teve efeito marginal total de -0,262, indicando que investimentos em educação reduzem as chances da aparição de focos de doenças associadas ao saneamento. A educação é, portanto, um importante objeto de políticas públicas, já que tem impactos na diminuição de infecções adquiridas por veiculação hídrica, como verminoses e as transmitidas por mosquitos. A existência de ouvidoria para receber denúncias ou reclamações sobre o saneamento da cidade aumenta a probabilidade de endemia e epidemia em 7,1\%. Entretanto, esse resultado pode estar associado ao maior número de denúncias nas cidades que possuem o serviço.

As favelas, cortiços e loteamentos irregulares existentes na cidade aumentam em 9,8\% a chance de ocorrer patologias ligadas ao saneamento. A localização dessas moradias pode induzir a incidência de doenças como malária e dengue. Assim como a estrutura das habitações pode influenciar na ocorrência de enfermidades feco-orais e provenientes da má higienização. Além disso, essas áreas contribuem para a proliferação de diversos vetores de doenças infectocontagiosas, como ratos, insetos e animais domésticos.

Dos quatro impactos ambientais observados nos últimos 24 meses, que foram testados no modelo, três foram significativos. 0 assoreamento e a diminuição de vazão de algum corpo d'água aumentam as chances de endemias e epidemias em 3,8\% e 3,6\%, respectivamente. Já a destinação inadequada do esgoto aumenta a probabilidade de ocorrência em 5,23\%. Sobre este último impacto cabe destacar que o esgotamento 
sanitário adequado é um dos principais instrumentos para a diminuição da morbidade hospitalar e da mortalidade em várias regiões do país e do mundo.

As análises regionais deste estudo foram feitas em relação à região Centro-0este do país. As cidades da região Nordeste têm 18,0\% a mais de chance de reportar endemias e/ou epidemias, e as cidades da região Norte têm 12,0\% a mais. A região Sudeste não foi estatisticamente diferente da Centro-Oeste. Por outro lado, a região Sul tem 20,5\% a menos de probabilidade de apresentar focos de doenças associadas ao saneamento. Os resultados mostram que os municípios de regiões mais ao centro da Zona Tropical têm maiores chances de ocorrência de patologias ligadas ao saneamento, enquanto as mais próximas da Zona Temperada têm suas probabilidades reduzidas. As diferentes características dos ecossistemas podem ser entendidas como fatores epidemiológicos, uma vez que podem ser habitats naturais para patógenos, vetores ou hospedeiros, a ocupação humana desses locais pode levar a focos naturais de doenças. Além disso, as diferentes organizações dos espaços geográficos dessas regiões podem contribuir para o aumento ou diminuição das enfermidades relacionadas ao saneamento (Silva, 1997).

No passo seguinte, foi verificado se a PMSB possuía algum padrão de aglomeração. Foram utilizados indicadores LISA para identificar quatro tipos de clusters: $\mathrm{HH}$, tanto o município quanto os vizinhos possuem a política; HL, apenas o município adotou a política, mas os vizinhos não; LH, o município não tem PMSB, mas os vizinhos têm; e LL, nem o município e nem o vizinho adotaram a política. Os clusters municipais estão apresentados no mapa da Figura 3.

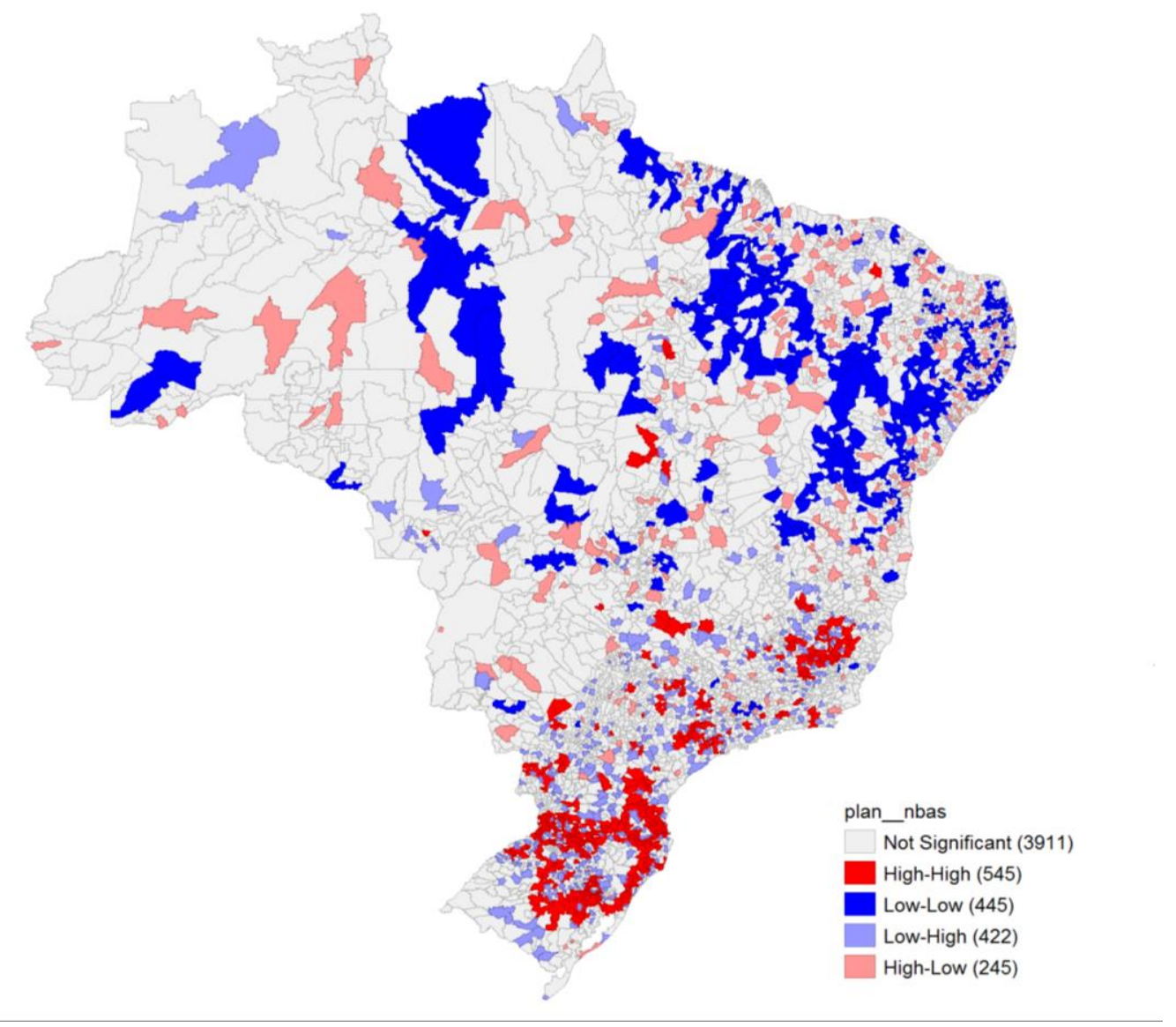

Figura 3 - Mapa dos clusters LISA para a existência de PMSB nos municípios brasileiros. Fonte: Elaboração própria.

Constatada a dependência espacial pela existência dos agrupamentos, coube investigar as chances do município que possui PMSB ter endemias e epidemias relacionadas ao saneamento inadequado considerando as circunstâncias no seu entorno (municípios vizinhos). Então, foram estimados modelos logit explicativos dos clusters, de municípios vizinhos com e sem a política de saneamento. A Tabela 5 apresenta os modelos para os quatro tipos de clusters. 
Tabela 5 - Modelos logit utilizando as aglomerações estimadas pelo LISA - 2017

\begin{tabular}{|c|c|c|c|c|c|c|c|c|}
\hline & $\mathrm{HH}$ & & HL & & LH & & LL & \\
\hline Variáveis & Coeficiente & $\operatorname{Pr}(>|z|)$ & Coeficiente & $\operatorname{Pr}(>|z|)$ & Coeficiente & $\operatorname{Pr}(>|z|)$ & Coeficiente & $\operatorname{Pr}(>|z|)$ \\
\hline Intercept & $-12,591$ & $* * *$ & $-1,219$ & & $-9,156$ & $* * *$ & 7,284 & $* * *$ \\
\hline end_epi & $-0,367$ & $* * *$ & 0,193 & & $-0,233$ & $*$ & 0,054 & \\
\hline $\log ($ dens17) & 0,488 & $* * *$ & 0,050 & & 0,133 & $* * *$ & $-0,092$ & $*$ \\
\hline txurbl6 & $-2,256$ & $* * *$ & 0,885 & $*$ & $-1,114$ & $* * *$ & 1,043 & $* *$ \\
\hline ifdml6educ & 3,308 & $* * *$ & $-3,048$ & $* * *$ & 2,893 & $* * *$ & $-2,613$ & $* * *$ \\
\hline $\log ($ pibpc 16 ) & 0,467 & $* * *$ & $-0,035$ & & 0,407 & $* * *$ & $-0,909$ & $* * *$ \\
\hline I(REGNAT) & 0,801 & $* * *$ & $-0,268$ & $* *$ & 0,184 & $* * *$ & $-0,142$ & \\
\hline
\end{tabular}

Fonte: Elaboração própria. Obs: ${ }^{*} p=0,10,{ }^{* *} p=0,05,{ }^{* * *} p=0,01$.

Quando um município possui a política de saneamento e está cercado por municípios que também detém tal política $(\mathrm{HH})$, as chances de ter doenças diminuem. 0 mesmo acontece quando um município não tem a política, mas é circundado por municípios que possuem a política de saneamento básico (LH). Entretanto, para os municípios em que nem todos os vizinhos possuem a PMSB (HL e LL), a variável que mede as endemias e epidemias relacionadas ao saneamento não teve efeito. Estes resultados confirmam a importância da dependência espacial e mostram que a PMSB diminui a chance de os municípios terem doenças, desde que não sejam casos isolados em termos geográficos.

\section{Considerações finais}

O saneamento básico se mostra um importante instrumento para o desenvolvimento econômico e para a garantia da qualidade do meio ambiente, evitando a ocorrência de impactos ambientais, assim como a diminuição de incidências de doenças decorrentes das condições precárias do saneamento. Diante de tal relevância, foram elaboradas políticas federais e municipais visando a universalização do acesso ao abastecimento de água, de esgotamento sanitário, manejo de águas pluviais e de resíduos sólidos, a fim de solucionar as mazelas enfrentadas pela sociedade.

O objetivo deste trabalho foi analisar o efeito que a PMSB exerce sobre a ocorrência das endemias e epidemias de doenças associadas aos serviços de saneamento nos municípios brasileiros. Para isso, foram estimados quatros modelos probit, por se tratar de variáveis binárias, sendo dois deles espaciais, com intuito de testar o efeito da correlação espacial entre os vizinhos na ocorrência de endemias e epidemias nos municípios do país.

Os resultados apontaram que ter a PMSB não apresenta efeito na redução de ocorrência de epidemias. Possivelmente, além das condições inapropriadas de saneamento, possam existir fatores relacionados às questões culturais, climáticas, geográficas que não foram abordadas neste trabalho e foram assimiladas pela presença das variáveis das Grandes Regiões. Outra suposição levantada é que apenas a existência da PMSB não tenha efeito, pois uma vez adotada seria necessária a implementação de aspectos específicos da política, tais como o Plano de Saneamento, o Conselho Municipal de Meio Ambiente, o fundo de Meio Ambiente, a legislação ambiental e os instrumentos de gestão.

A variável de educação, conforme explorado na literatura, reduz a incidência de surtos, pois quanto mais educadas as pessoas forem, melhores serão as boas práticas sanitárias, cuidados com a higiene e a contenção na proliferação de vetores. Ter acesso à ouvidoria ou à central de atendimento para reclamações e denúncias sobre saneamento aumenta os casos de epidemia e endemia nos municípios brasileiros, supostamente por ser um canal de comunicação entre a comunidade e as prestadoras dos serviços de saneamento, o que aumenta o número de casos reportados. A existência de favelas, cortiços e loteamento irregulares impacta positivamente nos casos de endemia e epidemia nas cidades. Vale ressaltar que a estrutura e condições deste tipo de moradias são propícias para a incidência de tais patologias. 0 impacto ambiental de assoreamento, diminuição de vazão de corpo d'água e a destinação inadequada do esgoto se 
mostraram fatores condicionantes na predominância de surtos, pois há muitas doenças relacionadas a estes problemas.

Ao analisar as aglomerações (clusters) dos municípios que possuem ou não a PMSB, foi constado que a política tem efeito quando toda a região no entorno da cidade possui a política. Estes resultados são importantes para se pensar a implementação da política municipal de saneamento. Tratá-la como uma política pública local parece não ser suficiente para mitigar a ocorrência de endemias e epidemias de doenças associadas ao saneamento básico, deve-se pensá-la e planejá-la para níveis geográficos mais abrangentes que os limites municipais.

\section{Referências}

Anselin, L. (1995). Local Indicators of Spatial Association-LISA. Geographical Analysis, 27(2), 93-115.

Anselin, L. (1988). Spatial econometrics: Methods and models. Springer Science \& Business Media, Berlim/Alemanha.

Araújo, A. F. V., Lima, J. C. M., Paixão, A. N. da, \& Picanço, A. P. (2010). Avaliação da Eficiência dos Serviços de Saneamento Básico no Combate às Endemias nos Municípios do Estado do Tocantins. Informe Gepec, 14, 166-181.

Borja, P. C. (2014). Política pública de saneamento básico: Uma análise da recente experiência brasileira. Saude e Sociedade, 23(2), 432-447.

Bradley, A. P. (1997). The use of the area under the ROC curve in the evaluation of machine learning algorithms. Pattern Recognition, 30(7), 1145-1159. https://doi.org/10.1016/S0031-3203(96)00142-2

Brasil (2007, 05 de janeiro). Lei $n^{\circ} 11.445$ de 05 de janeiro de 2007. Estabelece as diretrizes nacionais para o saneamento básico e para a política federal de saneamento básico. Brasília: Diário Oficial da União.

Câmara, G., Carvalho, M. S., Cruz, O. G., \& Correa, V. (2002). Análise de Dados de Área. In S. Druck, M. S. Carvalho, G. Câmara, \& A. M. V. Monteiro (Eds.), Análise Espacial de Dados Geográficos. Brasília: EMBRAPA. Recuperado em 7 de setembro de 2018. http://www.dpi.inpe.br/gilberto/livro/analise

Cairncross, S., \& Feachem, R. (1993). Environmental Health Engineering in the Tropics: an introductory text. Chichester : Wiley.

Cutler, D., \& Miller, G. (2005). The Role of Public Health Improvements in Health Advances: The 20th Century United States. Demography, 42 (1), 1-22. Recuperado em 11 de agosto de 2018, de https://www.jstor.org/stable/1515174.

Menezes, T. A., \& Uchoa, F. (2011). Mortalidade infantil, saneamento básico e o impacto da saúde sobre o crescimento econômico brasileiro. In Anais do XXXIX Encontro Nacional de Economia (p. 1-16). Foz do Iguaçu/Paraná: ANPEC.

Esrey, S. A., \& Habicht, J.-P. (1986). Epidemiologic evidence for health benefits from improved water and sanitation in developing countries. Epidemiologic Reviews, 8, 117-128.

Federação das Indústrias do Estado do Rio de Janeiro - FIRJAN. (2016). Índice FIRJAN de Desenvolvimento Municipal (IFDM) - 2016. Rio de Janeiro: IBGE. Recuperado em 13 de março de 2018, de https://www.firjan.com.br/ifdm/

Freitas, C. M. de. (2003). Problemas ambientais, saúde coletiva e ciências sociais. Ciência \& Saúde Coletiva, 8(1), 137-150.

Fundação Nacional de Saúde - Funasa (2013). $1^{\circ}$ caderno de pesquisa em engenharia da saúde pública. In Estudos e Pesquisas. Brasília: Ministério da Saúde, 244 p. Recuperado em 8 de agosto de 2018, de http://www.funasa.gov.br/bibliotecaeletronica/publicacoes/estudos-e-pesquisas1/-/asset_publisher/qGiy9skHw4ar/content/1-caderno-de-pesquisa-emengenharia-de-saude-publica?inheritRedirect=false.

Fundação Nacional de Saúde - Funasa (2014). Política e plano municipal de saneamento básico. In Convênio Funasa/Assemae. Brasília: Ministério da Saúde, 188 p. Recuperado em 8 de agosto de 2018, de http://www.funasa.gov.br/bibliotecaeletronica/publicacoes/engenharia-de-saude-publica/-/asset_publisher/ZM23z1KP6s6q/content/politica-e-planomunicipal-de-saneamento-basico-convenio-funasa-assemae?inheritRedirect=false.

Getis, A., \& Ord, J. K. (1992). The Analysis of Spatial Association by Use of Distance Statistics. Geographical Analysis, 24(3), 189-206. 
Getis, A., \& Ord, J. K. (1996). Local spatial statistics: an overview. In: P. Longley, P. \& Batty, M. (eds). Spatial analysis: modelling in a GIS environment (p. 261-277). New York: John Wiley \& Sons.

Gujarati, D. (2006). Econometria Básica (4 ed.). Elsevier.

Heller, L. (1998). Relação entre saúde e saneamento na perspectiva do desenvolvimento. Ciência \& Saúde Coletiva, 3 (2), $73-$ 84.

Instituto Brasileiro de Geografia e Estatística - IBGE. (2016a). Estimativas Populacionais - 2016. Rio de Janeiro: IBGE. Recuperado em 13 de março de 2018, de www.ibge.gov.br

Instituto Brasileiro de Geografia e Estatística - IBGE. (2017a). Estimativas Populacionais - 2017. Rio de Janeiro: IBGE. Recuperado em 13 de março de 2018, de www.ibge.gov.br

Instituto Brasileiro de Geografia e Estatística - IBGE. (2017b). Pesquisa de Informações Básicas Municipais (MUNIC) - 2017. Rio de Janeiro: IBGE. Recuperado em 11 de março de 2018, de www.ibge.gov.br

Instituto Brasileiro de Geografia e Estatística - IBGE. (2016b). Pesquisa Nacional por Amostra de Domicílios: Síntese de indicadores 2015. Rio de Janeiro: IBGE. Recuperado em 18 de junho de 2018, de www.ibge.gov.br.

Leoneti, A. B., Prado, E. L. do, \& Oliveira, S. V. W. B. de. (2011). Saneamento básico no Brasil: considerações sobre investimentos e sustentabilidade para o século XXI. Revista de Administração Pública, 45 (2), $331-348$. https://doi.org/10.1590/s0034-76122011000200003.

Lopez, D., \& Manogaran, G. (2017). Parametric Model to Predict H1N1 Influenza in Vellore District, Tamil Nadu, India. In: A. Rao, S. Pyne \& C. Rao (Eds.), Handbook of Statistics. (1ํㅡㄹ ed., Vol. 37), p.301-316.

Martinetti, D., \& Geniaux, G. (2017). Approximate likelihood estimation of spatial probit models. Regional Science and Urban Economics, 64, 30-45. https://doi.org/10.1016/j.regsciurbeco.2017.02.002

Mendonça, M., \& Motta, R. (2007). Saúde e Saneamento no Brasil. In Planejamento e Políticas Públicas (Vol. 30).0kawara, J. M. (2018). Avaliação de impacto da política de resíduos sólidos na saúde (Dissertação de Mestrado). Escola de Economia de São Paulo, Fundação Getulio Vargas - FGV, São Paulo.

Okawara, J. M. (2018). Avaliação de impacto da política de resíduos sólidos na saúde (Dissertação de Mestrado). Escola de Economia de São Paulo, Fundação Getúlio Vargas - FGV, São Paulo.

Organização das Nações Unidas - ONU. (2015). Transformando Nosso Mundo: A Agenda 2030 para o Desenvolvimento Sustentável. Nova YorK: ONU. Recuperado em 10 de maio de 2018, de www.undp.org/content/dam/brazil/Agenda2030completo-site (1).pdf

Paiva, R. F. da P. de S., \& Souza, M. F. da P. de. (2018). Associação entre condições socioeconômicas, sanitárias e de atenção básica e a morbidade hospitalar por doenças de veiculação hídrica no Brasil. Cadernos de Saúde Pública, 34 (1), 1-11. https://doi.org/10.1590/0102-311X00017316.

Rood, E. J. J., Goris, M. G. A., Pijnacker, R., Bakker, M. I., \& Hartskeerl, R. A. (2017). Environmental risk of leptospirosis infections in the Netherlands: Spatial modelling of environmental risk factors of leptospirosis in the Netherlands. PLoS ONE, 12 (10), 1-11. https://doi.org/10.1371/journal.pone.0186987.

Scriptore, J. S. (2010). A parceria público-privada no saneamento básico brasileiro: uma proposta para o desenvolvimento do setor (Dissertação de Mestrado). Faculdade de Economia, Administração e Contabilidade de Ribeirão Preto, Universidade de São Paulo - USP, Ribeirão Preto.

Silva, L. J. da. (1997). O conceito de espaço na epidemiologia das doenças infecciosas. Cadernos de Saúde Pública, 13 (4), 585593. https://doi.org/10.1590/s0102-311x1997000400002.

Silva, V. A., \& Esperidião, F. (2017). Saneamento básico e seus impactos na mortalidade infantil e no desenvolvimento econômico da região Nordeste. Scientia Plena, 13 (10), 1-7. https://doi.org/10.14808/sci.plena.2017.109905.

Siqueira, M. M., \& Moraes, M. S. de. (2009). Saúde coletiva, resíduos sólidos urbanos e os catadores de lixo. Ciência \& Saúde Coletiva, 14 (6), 2115-2122. https://doi.org/10.1590/S1413-81232009000600018.

Teixeira, J. C., Oliveira, G. S. de, Viali, A. de M., \& Muniz, S. S. (2014). Estudo do impacto das deficiências de saneamento básico sobre a saúde pública no Brasil no período de 2001 a 2009. Engenharia Sanitaria e Ambiental, 19 (1), 87-96. https://doi.org/10.1590/s1413-41522014000100010. 
Teixeira, L. (2011). Evidências empíricas das políticas de saneamento básico sobre indicadores de saúde para municípios brasileiros (Dissertação de Mestrado). Escola de Economia de São Paulo, Fundação Getúlio Vargas - FGV, São Paulo,.

Tipayamongkholgul, M. (2013). Spatial Analysis of Social Determinants for Tuberculosis in Thailand. J Med Assoc Thai, 96 (5), 116-121.

Turolla, F. A. (1999). Provisão e operação de infraestrutura no Brasil: o setor de saneamento. (Dissertação de Mestrado). Escola de Administração de Empresas, Fundação Getúlio Vargas - FGV, São Paulo.

Uhr, J. G. Z., Schmechel, M., \& Uhr, D. D. A. P. (2016). Relação entre saneamento básico no Brasil e saúde da população sob a ótica das internações hospitalares por doenças de veiculação hídrica. Revista de Administração, Contabilidade e Economia da Fundace, 7 (2), 1-16. https://doi.org/10.13059/racef.v7i2.104.

Venson, A. H., Rodrigues, K. C. T. T., \& Camara, M. R. G. da. (2017). Evolução da distribuição espacial do acesso aos serviços de saneamento básico nos municípios do Estado da Bahia, nos anos de 2006 e 2012. Ensaios FEE, Porto Alegre, 38, 107-134.

Vieira, G. B. (2017). Saúde Pública e Meio Ambiente: uma correlação com o saneamento básico. (Trabalho de Conclusão de MBA). Universidade Federal do Paraná, Curitiba.

Wilhelm, S., \& Godinho De Matos, M. (2013). Estimating Spatial Probit Models in R. The RJournal, 5 (1), 130-143. Recuperado em 21 de outubro de 2018 http://rjournal.github.io/

Wooldridge, J. M. (2014). Introdução à econometria: uma abordagem moderna (4a ed.). Cengage Learning.

World Health Organization - WHO (2018). Guidelines on sanitation and health. Recuperado em 16 de maio de 2018. https://www.who.int/water_sanitation_health/publications/guidelines-on-sanitation-and-health/en/

Editor: Harry Bollmann

Recebido: 20 dez. 2019

Aprovado: 27 out. 2020 